\title{
Phosphorous Containing Chitosan Beads for Controlled Oral Drug Delivery
}

\author{
R. JAYAKUMAR, R. L. REIS AND J. F. MANO* \\ $3 B$ 's Research Group-Biomaterials, Biodegradables and Biomimetics, \\ University of Minho, Campus of Gualtar, 4710-057 Braga, Portugal and \\ Department of Polymer Engineering, University of Minho, Campus of Azurem, \\ 4800-058 Guimaraes, Portugal
}

\begin{abstract}
Phosphorous containing chitosan (PCTS) was synthesized by a graft copolymerization technique in order to be used as controlled drug delivery devices. To test this potential, PCTS beads were prepared by using tripolyphosphate, at $\mathrm{pH} 4.0$ and characterized by scanning electron microscopy. The in vitro drug release behavior in various $\mathrm{pH}$ solutions was studied using indomethacin (IM) as a model drug at two different concentrations $(0.3$ and $0.6 \% \mathrm{w} / \mathrm{w})$. The release percent of IM from PCTS beads was found to be increased with the increasing of $\mathrm{pH}$ in the buffer solution. The release rate of IM at $\mathrm{pH} 7.4$ was higher than that at $\mathrm{pH}$ 1.4, due to the ionization of phosphorous groups and the high solubility of IM in the alkaline medium. These results indicated that PCTS beads have the potential to be used as controlled drug delivery systems through oral administration by avoiding the drug release in the highly acidic gastric fluid region of the stomach.
\end{abstract}

KEY WORDS: modified chitosan, chitosan beads, indomethacin, drug delivery, $\mathrm{pH}$ sensitive drug delivery, oral drug delivery.

\section{INTRODUCTION}

Chitosan (CS) is a biopolymer comprised of D-glucosamine and D-acetyl-glucosamine units that can be readily processed into films, porous scaffold, beads and fibers from aqueous acid solution. CS

*Author to whom correspondence should be addressed.

E-mail: jmano@dep.uminho.pt

Figures 2, 3, 4, 5, 6, 7 and 8 appear in color online: http://jbc.sagepub.com

Journal of Bioactive and Compatible Polymers, Vol. 21-July 2006 
beads have been developed for controlled drug release and removal of heavy metal ions from waste water [1-3]. CS displays interesting properties such as biocompatibility, biodegradability [4] and its degradation products are non-toxic, non-immunogenic and non-carcinogenic [5].

Recently, there has been a growing interest in studying the chemical modification of CS to improve its solubility and widen its applications [6-10]. Among the various methods, graft copolymerization is the most attractive because it is a useful technique for modifying chemical and physical properties of natural polymers. CS has both primary amine and alcohol reactive groups that can be grafted. Covalent grafting of molecule entities allows the formation of functional derivatives onto CS backbone with desired chemical groups. It has been shown that, after primary deviation followed by graft modification, CS improved water solubility and bioactivities such as antibacterial and antioxidant properties [11-13], chelating [14] or complexation properties [15], bacteriostatic effect [16] or enhancing adsorption properties [17,18]. Although the grafting of CS modifies its properties, it is possible to maintain some interesting characteristics such as mucoadhesivity [19], biocompatibility [20-22] and biodegradability [23]. Many investigations have been carried out on the graft copolymerization of CS [13-22] with a view to preparing polysaccharide-based advanced materials with unique properties and thus widening their applications in biomedical and environmental fields.

CS is a very promising biomaterial for drug delivery as it readily responds to $\mathrm{pH}$. However, the use of soluble CS polymer in oral administration is restricted by its fast dissolution in the stomach and limited capacity for a controlled drug delivery system [24]. In order to overcome these disadvantages, tripolyphosphate (TPP)/CS polyelectrolyte complexes as gel beads were developed for sustained release performances [25-28]. PCTS has attracted considerable interest because among its advantages, it exhibits anti-inflammatory properties and it forms both metal complexes and anionic polyelectrolyte hydrogels [29-33]. In this work, CS was grafted with TPP to prepare beads in order to improve the sustained release system in a gastric fluid. By using 1-ethyl-3-(3-dimethylaminopropyl) carbodiimide (EDC) mediated coupling reaction, 2-carboxethyl phosphonic acid was covalently grafted onto chitosan. The morphology, swelling properties and in vitro drug profiles of the prepared beads were evaluated to obtain an insight to the potential of this system for $\mathrm{pH}$ sensitive controlled release of drugs or other bioactive molecules. 


\section{EXPERIMENTAL}

\section{Materials}

Chitosan (CS) (deacetylation degree 75-85\%), tripolyphosphate (TPP) and 2-carboxyethylphosphonic acid were purchased from Aldrich. EDC was obtained from Alfa Aesar Company. IM was purchased from Fluka. All other materials used were of analytical grade.

\section{Synthesis of Phosphorous Containing Chitosan (PCTS)}

PCTS was prepared according to the method reported in [32]. Briefly, CS $(0.9712 \mathrm{~g})$ was dissolved in $10 \mathrm{~mL}$ of $1 \% \mathrm{w} / \mathrm{w}$ acetic $\mathrm{acid} / \mathrm{methanol}$ solution and the $\mathrm{pH}$ solution was then raised to 5.5 by adding $1 \mathrm{M} \mathrm{NaOH}$. 2-Carboxyethylphosphonic acid $(0.4621 \mathrm{~g})$ was dissolved in $10 \mathrm{~mL}$ of water and then added drop by dropwise to the CS solution. To this mixture EDC $(0.4552 \mathrm{~g})$ was added. The reaction was carried out at $70^{\circ} \mathrm{C}$ for $7 \mathrm{~h}$. The reaction mixture was filtered and affluent dialyzed against spectropor membrane tubing ( $\mathrm{Mw}$ cut-off 3500 ) for 3 days with 10 changes of water to eliminate the impurities. The resulting solution was precipitated with acetone and dried at room temperature.

\section{Preparation of Phosphorous Containing Chitosan (PCTS) Beads}

PCTS beads were prepared using an ionotropic gelation process with the counter polyanion TPP. PCTS $(0.7512 \mathrm{~g})$ was dissolved in $10 \mathrm{~mL}$ of $2 \% \mathrm{w} / \mathrm{w}$ acetic acid solution and stirred for $24 \mathrm{~h}$ to obtain a transparent homogeneous solution. The PCTS solution with or without drug was introduced into a stirred 10\% TPP aqueous phase using a syringe needle $(0.5 \mathrm{~mm}$ in diameter $)$; solid white beads were formed immediately. These beads were allowed to stand for $1 \mathrm{~h}$ in the TPP solution with occasional agitation at room temperature and $\mathrm{pH}$ 4.0. The gel beads were filtered, washed with distilled water repeatedly and dried under vacuum at room temperature for 2 days. For the preparation of drug loaded PCTS beads, two different amounts of IM $(0.3$ and $0.6 \% \mathrm{w} / \mathrm{w}$ with respect to PCTS solution) were dispersed in $10 \mathrm{~mL}$ of PCTS solution for $24 \mathrm{~h}$. The same procedure was followed for the isolation and purification as described above. 


\section{Swelling Evaluation}

The dried CS and PCTS beads were carefully weighed and immersed in to $50 \mathrm{~mL}$ of buffer solutions of $\mathrm{pH} 1.4$ and 7.4 at $37^{\circ} \mathrm{C}$. At predetermined time intervals, swollen beads were taken out, and the excess water was blotted with filter paper from the surface, and then weighed. The following equation was used to determine the swelling degree:

$$
\text { Swelling degree }[\%]=\left\{\left(X_{w}-X_{d}\right) / X_{d}\right\} \times 100
$$

where $X_{d}$ and $X_{w}$ represent the mass of dry and swollen beads, respectively.

\section{Determination of Drug Loading Efficiency}

During the gelation process in TPP aqueous solution, some IM diffused from PCTS droplet into TPP solution. After gelation, the IM concentration in the TPP solution was determined by a UV spectrophotometer at $320 \mathrm{~nm}$. The loading efficiency during the gelation process was calculated by the following formula:

$$
\text { Loading efficiency }[\%]=\{(m-c \times v) / m\} \times 100
$$

where $m, c$ and $v$ represent the initial IM mass in PCTS droplet, the IM concentrations in TPP solution and the volume of TPP solution, respectively.

\section{In Vitro Drug Release Studies}

In vitro release tests were carried out on all formulations of the drug-loaded CS beads and PCTS beads. Known amounts of drugloaded gel beads were suspended in $100 \mathrm{~mL}$ of various buffer solutions in different $\mathrm{pH}$ media (1.4, 5.8, 7.4 and 9.4, respectively), stirred at $120 \mathrm{rpm}$ and maintained at $37^{\circ} \mathrm{C}$ in a water bath. At predetermined time intervals, $1 \mathrm{~mL}$ aliquots were withdrawn and dilutioned to determine the concentration of drug released by UV spectrophotometry at $320 \mathrm{~nm}$. The dissolution medium was replaced with a fresh buffer to maintain the total volume. The drug release percent was determined in terms of percentage by the following equation:

$$
\text { Drug release }[\%]=R_{t} / L \times 100
$$


where, $L$ and $R_{t}$ represent the initial amount of drug loaded and the cumulative amount of drug released at time $t$.

\section{Characterization Studies}

The surface morphology of samples was analyzed by scanning electron microscopy (Leica Canbridge S360 microscope). The drug release was monitored by UV spectrophotometry (Shimadzu UV-1601, Japan). The IR spectrum was recorded with a double-beam Perkin-Elmer 1600 FTIR spectrophotometer.

\section{RESULTS AND DISCUSSION}

\section{Synthesis of Phosphorous Containing Chitosan (PCTS) Beads}

Chitosan (CS, a polycationic polysaccharide) forms gels with multivalent counter ions through the formation of intermolecular or intramolecular linkages by ionic interactions. The liquid-gel transition of PCTS in TPP aqueous solution is due to the electrostatic interactions between positively charged free amino groups with negatively charged counter anion TPP [24]. A structure of PCTS beads is shown in Figure 1.

The small molecule polyelectrolyte TPP dissociated in water and released $\mathrm{OH}^{-}$ions, thus providing both $\mathrm{OH}^{-}$and $\mathrm{P}_{3} \mathrm{O}_{10}{ }^{5-}$ ions which coexisted in the TPP solution. The $\mathrm{OH}^{-}$and $\mathrm{P}_{3} \mathrm{O}_{10}{ }^{5-}$ ions could ionically react with the $-\mathrm{NH}_{3}{ }^{+}$binding site on PCTS by deprotonation and crosslinking, respectively. The CS-TPP complex formation in the gel beads was less time dependant when the outer layer was formed. The CS-phosphoric acid complexation gel beads were found to be time dependant [34]. Consequently, for the PCTS beads, the electrostatic attraction would be dependant on the residual $-\mathrm{NH}_{2}$.

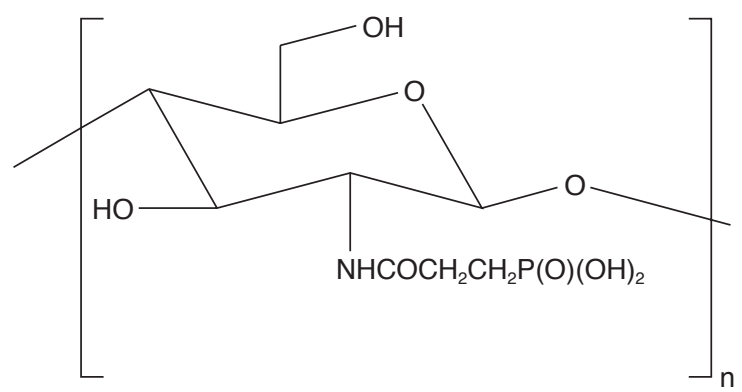

Figure 1. Structure of PCTS. 


\section{SEM of Beads}

The SEM of the CS and PCTS beads is shown in Figure 2; based on these SEM images the size of the CS and PCTS beads varied from 1.5 to $1.75 \mathrm{~mm}$. The CS beads exhibited a smooth surface morphology as well as a more regular spherical geometry and smooth surface than the PCTS beads. This may be due to a more efficient crosslinking in the CS beads. The PCTS beads exhibited some fissures on the surface that may be due to the grafting of phosphorous containing monomer. Crosslinking of PCTS beads was less efficient; some of the amino groups of CS were substituted by phosphorous which led to the formation of a rougher and non-uniform surface structure.

\section{Swelling Behavior of Beads}

The swelling behavior of the CS and PCTS beads in $\mathrm{pH} 1.4$ and 7.4 solutions at $37^{\circ} \mathrm{C}$ are shown in Figures 3 and 4 . The PCTS beads at $\mathrm{pH}$ 1.4 disintegrated within 3 days and then dissolved while the CS beads dissolved after 4 days. These results showed that the swelling of the CS-TPP beads was different from that of PCTS beads agreement with the difference in their crosslinking density. The less crosslinked PCTS beads swelled quickly and gradually dissolved within 3 days, whereas the highly crosslinked CS beads did not dissolve within the same time period. This was expected behavior, due to the high amount of interchain linkages and masking of the hydrophilic nature of the amino groups in the high ionic crosslinked CS-TPP network [35]. The swelling percentage was found to be much higher at $\mathrm{pH} 1.4$ than $\mathrm{pH}$ 7.4. Moreover, the swelling reached a stable equilibrium more rapidly

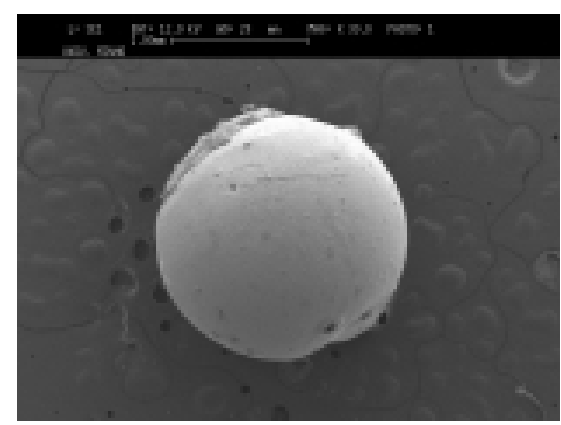

(a)

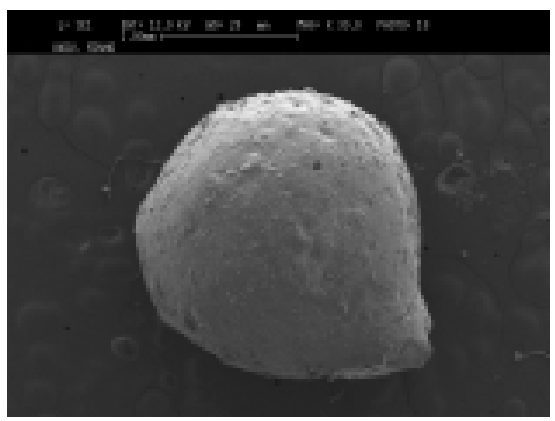

(b)

Figure 2. SEM of the beads (a) CS and (b) PCTS. Scale bars $=1 \mathrm{~mm}$ 


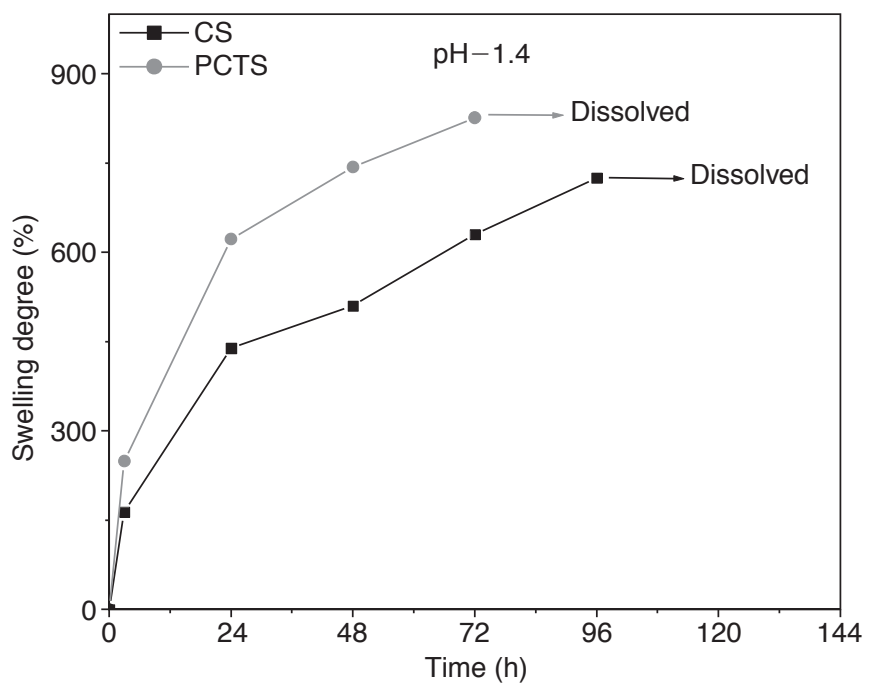

Figure 3. Swelling behavior of the CS and the PCTS beads at pH 1.4.

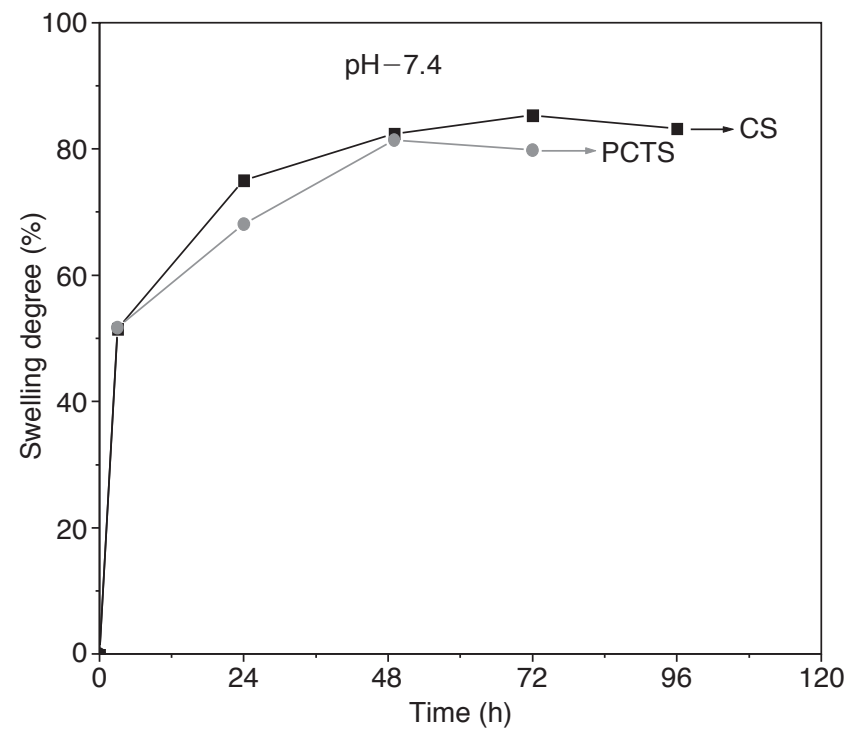

Figure 4. Swelling behavior of the CS and the PCTS beads at pH 7.4. 
at $\mathrm{pH} 7.4$ than at $\mathrm{pH}$ 1.4. This was due to the lack of protonation of amino groups at $\mathrm{pH}$ 7.4, which prevents swelling in an alkaline medium [36].

\section{Entrapment of IM in PCTS Beads}

IM is a non-steroid anti-inflammatory drug used for the treatment of arthritis [33]. It was efficiently entrapped into both CS and PCTS beads during the ionotropic process (Table 1). The relatively high efficiency of loading may be due to the low water-solubility of IM as well as the ionic binding between negatively charged carboxyl groups in IM and positively charged free amino groups in CS. The carboxyl groups in IM and the phosphorous groups in PCTS are ionized at $\mathrm{pH}$ 4. In this work, the drug was not chemically attached to the polymer. Thus, the drug in the gel was only held by the electrostatic and hydrophobic interactions, and physical entrapment within the polymer matrix. Consequently, the drug remained in a biologically active form and therapeutically effective in the body as soon as it was released from the polymeric matrix.

\section{The In Vitro Release Profiles of IM}

The release profiles for $0.3 \%$ IM-PCTS beads at various $\mathrm{pH}$ 's and at $37^{\circ} \mathrm{C}$ in a buffer solution are shown in Figure 5. In general, the percent of drug released increased with increasing $\mathrm{pH}$ of medium. Within $3 \mathrm{~h}$, $30 \%$ of the IM was released from the PCTS beads at $\mathrm{pH} 1.4$ and $50 \%$ at $\mathrm{pH}$ 7.4. This behavior indicates that drug release profiles are $\mathrm{pH}$-sensitive. There are two factors to be considered in the noticeably higher release rate of IM at $\mathrm{pH} 7.4$ than at $\mathrm{pH}$ 1.4. The first factor is the high solubility of IM in alkaline medium and the other is the electrostatic repulsion between the negatively ionized carboxyl group of IM and the phosphate groups in PCTS at pH 7.4 [36-38]. Similar drug release profiles were observed for the CS beads. However, the release percentage

Table 1. Loading efficiencies for IM-loaded CS and PCTS beads.

\begin{tabular}{cccc}
\hline Formulation & $\begin{array}{c}\text { Initial Drug Content } \\
\text { (mg/g-beads) }\end{array}$ & $\begin{array}{c}\text { Loading Level } \\
\text { (mg/g-beads) }\end{array}$ & $\begin{array}{c}\text { Loading Efficiency } \\
\text { (\%) }\end{array}$ \\
\hline 0.3\% IM-CS & 28.9 & $27.11 \pm 0.5$ & $93.81 \pm 1.9$ \\
0.6\% IM-CS & 58.2 & $53.21 \pm 2.0$ & $91.43 \pm 3.8$ \\
0.3\% IM-PCTS & 28.9 & $25.17 \pm 0.3$ & $87.09 \pm 1.4$ \\
0.6\%IM-PCTS & 58.2 & $49.34 \pm 5.1$ & $84.78 \pm 5.3$ \\
\hline
\end{tabular}


of IM at $\mathrm{pH} 1.4$ was found to be less than that for the PCTS beads. The reason may be due to the formation of complexes between the carboxyl group of IM and the amino group of CS and poor solubility of the drug at $\mathrm{pH} 1.4$ [36-38]. The diffusion rate of the drug molecules was higher in the alkaline medium, effectively contributing to the controlled drug release. The polymer matrix probably combines two types of mechanisms for drug release: controlled diffusion and swelling. The in vitro release profiles of $0.6 \%$ IM-loaded CS and PCTS beads at $\mathrm{pH} 1.4$ and 7.4 are shown in Figure 6; similar release profiles were observed.

The influence of the initial drug loading on the release profiles of IM from PCTS beads at pH 1.4 and 7.4 is shown in Figure 7. Surprisingly, there was no significant difference between the release of IM for 0.3 and $0.6 \%$ loaded PCTS beads. For initial drug loadings, the amounts of IM released per unit mass of PCTS beads at $\mathrm{pH} 7.4$, are shown in Figure 8. As expected, the amount of drug released from highly loaded beads was higher than that from slightly loaded beads. Increasing the initial drug content provided the larger equilibrium amount of drug released at each $\mathrm{pH}$.

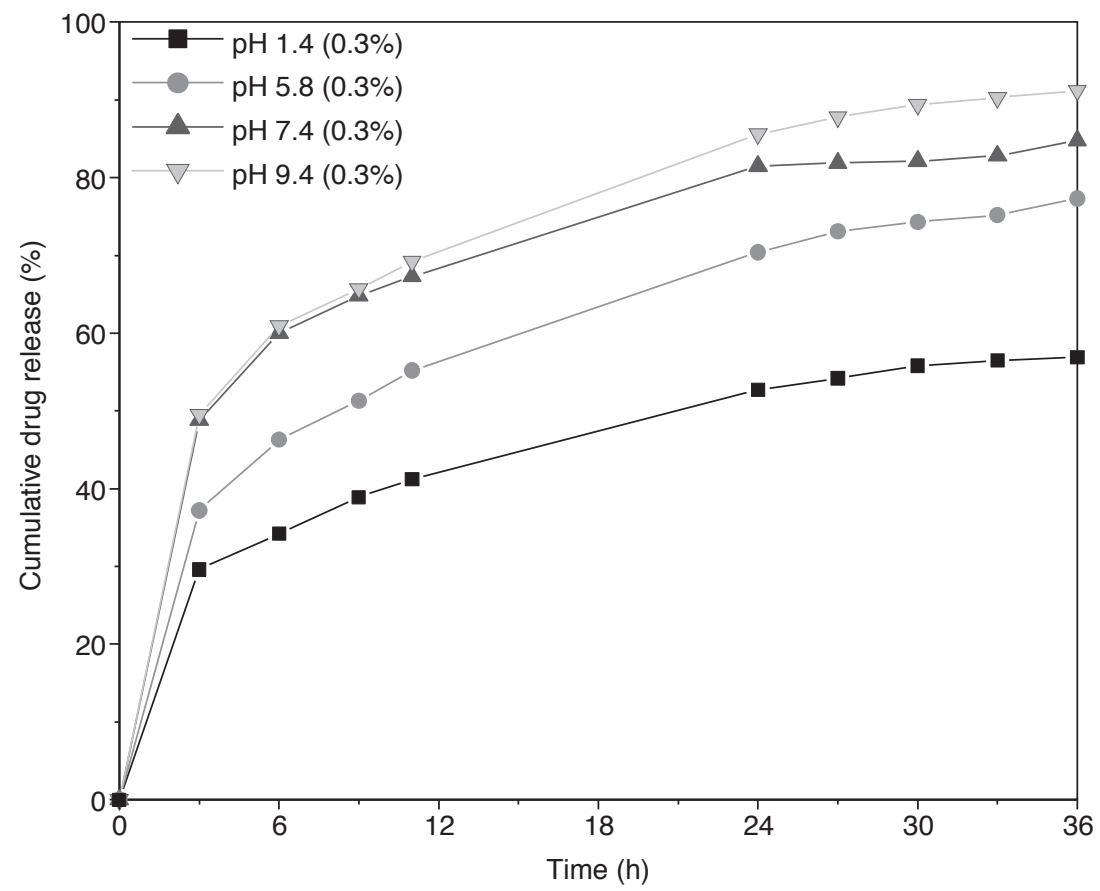

Figure 5. Release profiles of IM from $0.3 \%$ loaded PCTS beads in various $\mathrm{pH}$ media. 


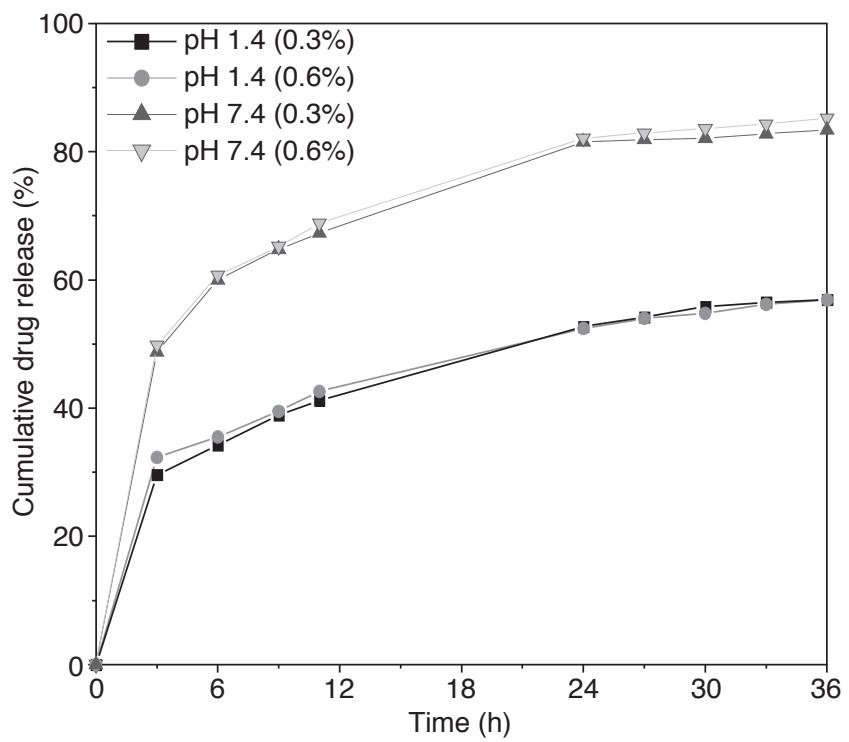

Figure 6. Drug release from IM-loaded PCTS beads with different initial drug contents $(0.3$ and $0.6 \%)$ in simulated gastric fluid, $\mathrm{pH} 1.4$, and simulated intestinal fluid, $\mathrm{pH} 7.4$.

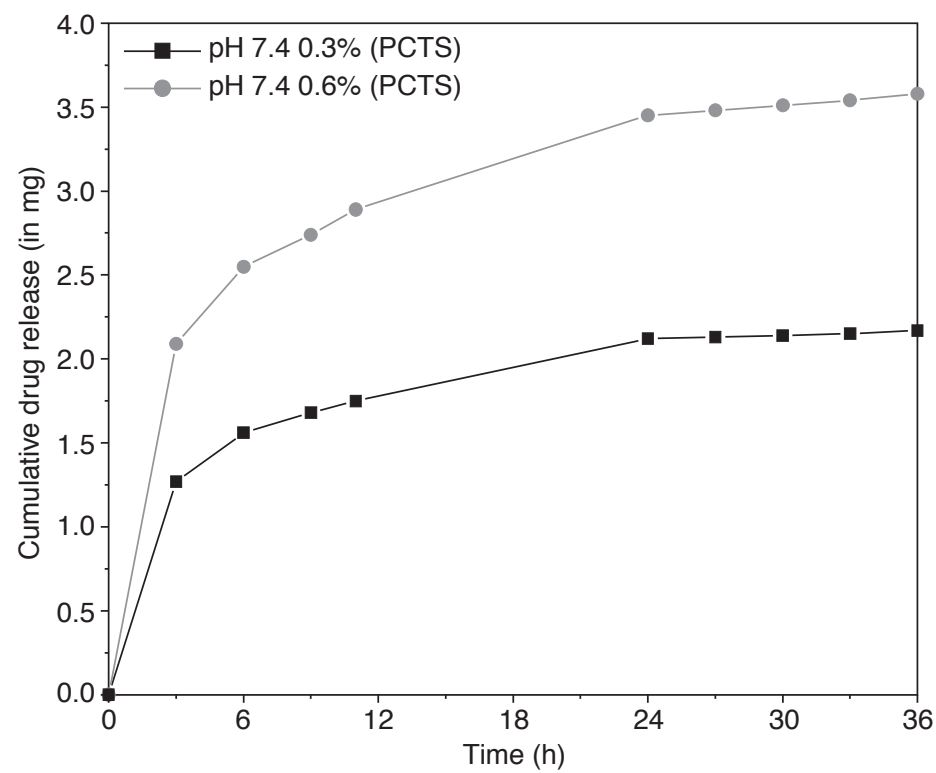

Figure 7. Time course of IM amount released from PCTS beads and IM concentration in dissolution buffer at different initial drug contents $(0.3$ and $0.6 \%)$ at $\mathrm{pH} 7.4$. 


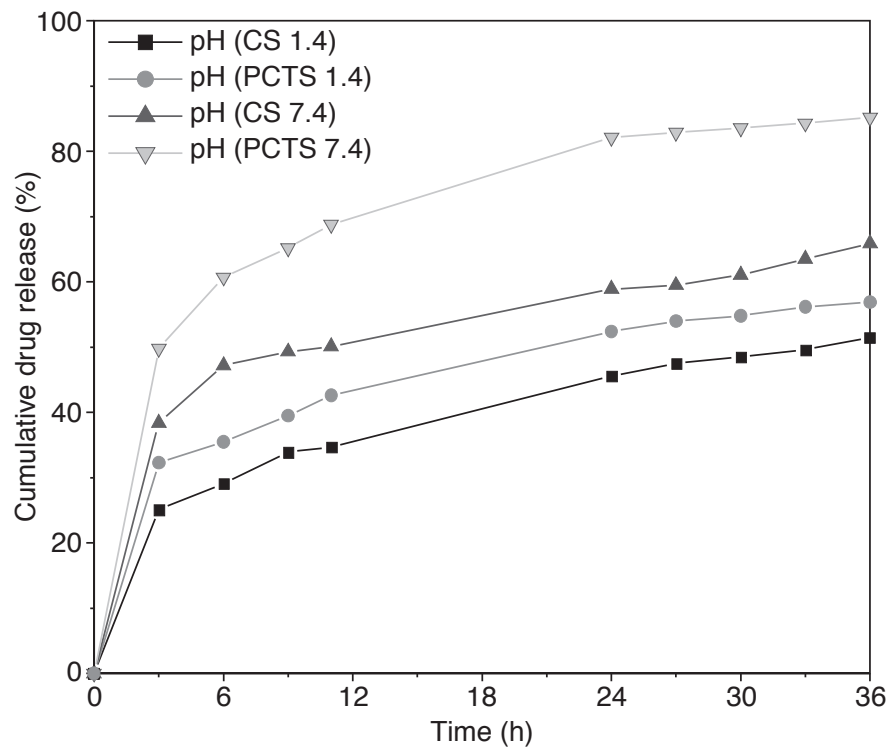

Figure 8. Release profiles for initially $0.6 \%$ IM-loaded CS and PCTS beads in simulated gastric fluid, $\mathrm{pH}$ 1.4, and simulated intestinal fluid, $\mathrm{pH}$ 7.4.

\section{CONCLUSIONS}

In this work, PCTS beads were developed in order to be used as a $\mathrm{pH}$ sensitive and gastric fluid-resistant drug carriers. The beads were prepared under mild conditions at room temperature and $\mathrm{pH} 4.0$, and the loading efficiency of the model drug, IM, was over $85 \%$. The water solubility of the drug, the swelling degrees of beads and the ionization of phosphate groups were found to have a significant influence on the release profiles of IM at various pHs. For example, the release rate from the PCTS beads in simulated intestinal fluid ( $\mathrm{pH}$ 7.4) was higher than that in simulated gastric fluid $(\mathrm{pH} 1.4)$. These factors suggest that the PCTS beads could be better than chitosan beads as a controlled drug delivery system for oral administration by avoiding drug release in the highly acidic gastric fluid region of the stomach.

\section{ACKNOWLEDGMENTS}

R. Jayakumar thanks the Portuguese Foundation for Science and Technology for providing him with a Post-Doctoral scholarship (SFRH/BPD/14670/2003). This work was partially supported by FCT 
Foundation for Science and Technology through funds from the POCTI and/or FEDER programmes. This work was partially supported by the European Union funded STREP Project HIPPOCRATES (NMP3-CT-2003-505758).

\section{REFERENCES}

1. Borchard, G. (2001). Chitosans for Gene Delivery, Advanced Drug Delivery Review, 52(1): 145-150.

2. Felt, O., Furrer, P., Mayer, J.M., Plazonnet, B., Buri, P. and Gurney, R. (1999). Topical Use of Chitosan in Ophthalmology: Tolerance Assessment and Evaluation of Precorneal Retention. International Journal of Pharmaceutics, 180: 185-193.

3. Prabaharan, M. and Mano, J.F. (2005). Chitosan-based Particles as Controlled Drug Delivery Systems, Drug delivery, 12(1): 41-57.

4. Ravi Kumar, M.N.V. (2001). A Review of Chitin and Chitosan Applications, Reactive and Functional Polymers, 46(1): 1-27.

5. Muzzarelli, R.A.A. (1997). Human Enzymatic Activities Related to the Therapeutic administration of Chitin Derivatives, Cell Molecular Life Science, 53(1): 131-140.

6. Sugimoto, M., Morimoto, M. and Sashiwa, H. (1998). Preparation and Characterization of Water-soluble Chitin and Chitosan Derivatives, Carbohydrate Polymers, 36(1): 49-59.

7. Hornof, M., Kast, C.E. and Schnurch, A.B. (2003). In vitro Evaluation of the Viscoelastic Behavior Chitosan-Thioglycolic Acid Conjugates, European Journal of Pharmaceutics and Biopharmaceutics, 55(2): 185-190.

8. Sashiwa, H. and Shigemasa, Y. (1999). Chemical Modification of Chitin and Chitosan 2: Preparation and Water Soluble Property of $N$-acylated or $N$-alkylated Partially Deacetylated Chitins, Carbohydrate Polymers, 39(1): 127-138.

9. Heras, A., Rodriguez, N.M. and Ramos, V.M. (2001). N-Methylene Phosphonic Chitosan: A Novel Soluble Derivative, Carbohydrate Polymers, 44(1): 1-8.

10. Prabaharan, M. and Mano, J.F. (2006). Chitosan Derivatives Bearing Cyclodextrin Cavities as Novel Adsorbent Matrices, Carbohydrate Polymers, 63(2): 153-166.

11. Xie, W.M., Xu, P.X., Wang, W. and Liu, Q. (2001). Antioxidant Activity of Water-Soluble Chitosan Derivatives, Biomaterial and Medical Chemistry Letters, 13: 1699-1701.

12. Xie, W.M., Xu, P.X., Wang, W. and Liu, Q. (2002). Preparation and Antibacterial Activity of a Water-soluble Chitosan Derivative, Carbohydrate Polymers, 50(1): 35-40.

13. Jayakumar, R., Prabaharan, M., Reis, R.L. and Mano, J.F. (2005). Graft Copolymerized Chitosan - Present Status and Applications, Carbohydrate Polymers, 62(2): 142-158. 
14. Yang, Z.K. and Yuan, Y. (2001). Studies on the Synthesis and Properties of Hydroxyl Azacrown Ether-grafted Chitosan, Journal of Applied Polymer Science, 82: 1838-1843.

15. Chen, S. and Wang, Y. (2001). Study on $\beta$-cyclodextrin Grafting with Chitosan and Slow Release of its Inclusion Complex with Radioactive Iodine, Journal of Applied Polymer Science, 82: 2414-2421.

16. Jung, B.O., Kim, C.H., Choi, K.S., Lee, Y.M. and Kim, J.J. (1999). Preparation of Amphiphilic Chitosan and their Antimicrobial Activities, Journal of Applied Polymer Science, 72: 1713-1719.

17. Kotze, A.F., Luessen, H.L., Leeuw, B.J.D., Boer, A.G.D., Verhoef, J.C. and Junginger, H.E. (1997). $N$-Trimethyl Chitosan Chloride as a Potential Absorption Enhancer Across Mucosal Surfaces: In Vitro Evaluation in Intestinal Epithelial Cells $\left(\mathrm{CaCO}_{2}\right)$, Pharmaceutical Research, 14: 1197-1202.

18. Thanou, M., Verhoef, J.C. and Junginger, H.E. (2001). Oral Drug Absorption Enhancement by Chitosan and its Derivatives, Advanced Drug Delivery Reviews, 52: 117-126.

19. Hoffman, A.S., Chen, G., Wu, X., Ding, Z., Kabra, B., Randeri, K., Schiller, M.R.E., Peppas, N.A. and Brazel, C. (1997). Graft Copolymers of PEOPPO-PEO Triblock Polyethers on Bioadhesive Polymer Backbones: Synthesis and Properties, Polymer Preprints, 38: 524-525.

20. Tasker, R.A., Connel, B.J., Ross, S.J. and Elson, C.M. (1998). Development of an Injectable Sustained-release Formulation of Morphine: Antinociceptive Properties in Rats, Laboratory Animals, 32: 270-275.

21. Ono, K., Saito, Y., Yura, H. and Ishikawa, K. (2000). Photocrosslinkable Chitosan as a Biological Adhesive, Journal of Biomedical Material Research, 49: 289-295.

22. Prabaharan, M. and Mano, J.F. (2005). Hydroxypropyl Chitosan Bearing $\beta$-cyclodextrin Cavities: Synthesis and Slow Release of its Inclusion Complex with a Model Hydrophobic Drug, Macromolecular Bioscience, 5: 965-973.

23. Singh, D.K. and Ray, A.R. (1998). Characterization of grafted chitosan films, Carbohydrate Polymers, 36: 251-255.

24. Risbud, M.V., Haridikar, A.A., Bhat, S.V. and Bhonde, R.R. (2000). pHSensitive Freeze-Dried Chitosan-Polyvinyl Pyrrolidone Hydrogels as Controlled Release System for Antibiotic Delivery, Journal of Controlled Release, 68(1): 23-30.

25. Ghanem, A. and Skonberg, D. (2002). Effect of Preparation Method on the Capture and Release of Biologically Active Molecules in Chitosan Gel Beads, Journal of Applied Polymer Science, 84(2): 405-413.

26. Mi, F.L., Shyu, S.S., Lee, S.T. and Wong, T.B. (1999). Kinetic Study of Chitosan-tripolyphosphate Complex Reaction and Acid-resistive Properties of the 
Chitosan-tripolyphosphate Gel Beads Prepared by In-liquid Curing Method, Journal of Polymer Science, Part-B, Polymer Physics, 37: 1551-1564.

27. Mi, F.L., Sung, H.W. and Shyu, S.S. (2002). Drug Release from Chitosanalginate Complex Beads Reinforced by a Naturally Occurring Crosslinking Agent, Carbohydrate Polymers, 48: 61-72.

28. Shu, X.Z. and Zhu, K.J. (2000). A Novel Approach to Prepare Tripolyphosphate/Chitosan Complex Beads for Controlled Release Drug Delivery, International Journal of Pharmaceutics, 201(1): 51-58.

29. Lee, Y.M. and Shin, E.M. (1991). Pervaporation Separation of Water-Ethanol through Modified Chitosan Membranes. IV. Phosphorylated Chitosan Membranes, Journal of Membrane Science, 64(1): 145-152.

30. Nishi, N., Ebina, A., Nishimura, S., Tsutsumi, A., Hasegawa, O. and Tokura, S. (1986). Highly Phosphorylated Derivatives of Chitin, Partially Deacetylated Chitin and Chitosan as New Functional Polymers: Preparation and Characterization, International Journal of Biological Macromolecules, 8(5): 311-317.

31. Win, P.P., Ya, Y.S., Hong, K.J. and Kajiuchi, T. (2003). Formulation and Characterization of $\mathrm{pH}$ Sensitive Drug Carrier Based on Phosphorylated Chitosan, Carbohydrate Polymers, 53(3): 305-310.

32. Baran, E.T., Jayakumar, R., Mano, J.F. and Reis, R.L. (2006). Enzymatic Degradation Behaviour of Starch Conjugated Phoshorylated Chitosan, Material Science Forum, 514-516: 995-999.

33. Mi, F.L., Shyu, S.S., Chen, C.T. and Lai, J.Y. (2002). Adsorption of Indomethacin onto Chemically Modified Chitosan Beads, Polymer, 43: 757-765.

34. Mi, F.L., Shyu, S.S., Kuan, C.Y., Lee, S.T., Lu, K.T. and Jang, S.F. (1999). Chitosan-polyelectrolyte Complexation for the Preparation of Gel Beads and Controlled Release of Anticancer Drug. I. Effect of Phosphorous Polyelectrolyte Complex and Enzymatic Hydrolysis of Polymer, Journal of Applied Polymer Science, 74(7): 1868-1879.

35. Lee, S.T., Mi, F.L., Shen, Y.J. and Shyu, S.S. (2001). Equilibrium and Kinetic Studies of Copper(II) Ion Uptake by Chitosan-tripolyphosphate Chelating Resin, Polymer, 42: 1879-1892.

36. Gupta, K.C. and Ravi Kumar, M.N.V. (2000). Semi-interpenetrating Polymer Network Beads of Crosslinked Chitosan-Glycine for Controlled Release of Chlorphenramine Maleate, Journal of Applied Polymer Science, 76(5): $672-683$.

37. Hadgraft, J. and Valenta, C. (2000). pH, pKa and Dermal Delivery, International Journal of Pharmaceutics, 200(2): 243-247.

38. Jayakumar, R., Reis, R.L. and Mano, J.F. (2006). Synthesis and Characterization of pH-Sensitive Thiol-containing Chitosan Beads for Controlled Drug Delivery Applications, Drug Delivery (in press). 